http://jmscr.igmpublication.org/home/

ISSN (e)-2347-176x ISSN (p) 2455-0450

crossref DOI: https://dx.doi.org/10.18535/jmscr/v7i11.137

Journal Of Medical Science And Clinical Research

\title{
Non-Alcoholic fatty liver disease and Diabetes Mellitus
}

\section{Dr Tameem Imran ${ }^{1}$, Dr Rumaisa Ahmed ${ }^{2}$, Dr Vishwanath Reddy ${ }^{3}$}

\begin{abstract}
Diabetes is a systemic disease that affects almost all organ systems. Apart from causing major macro \& microvascular complications Non-alcoholic fatty liver disease (NAFLD) is being increasingly recognized today as a potentially serious complication of diabetes, especially type 2.

The high incidence of non-alcoholic steatohepatitis (NASH) in patients with T2DM leads to further complications, such as liver cirrhosis and hepatocellular carcinoma, which are increasingly recognized. ${ }^{[4]}$

So it is of prime importance to screen for Non-alcoholic fatty liver disease (NAFLD) before it progresses to NASH.

This observational study was conducted at $R$ L Jalappa Hospital on 75 diabetics (both type 1 and type 2). Among the 75 diabetics who were studied, fatty liver was found in 32 patients (42.66\%). All the patients with fatty liver had central obesity. Overweight among the persons detected to have fatty liver - 25 (78.1\%).

NASH can exist with only nonspecific symptoms for years in obese patients before manifesting itself either incidentally or with complications of cirrhosis or portal hypertension.

NAFLD and NASH have been described in patients without the classic risk factors of obesity, diabetes, and overt hyperlipidemia. Hypertriglyceridemia was identified as an important risk factor in the development of NAFLD and NASH.

Conclusion: The prevalence of non-alcoholic fatty liver disease in type 2 diabetes mellitus was $42.6 \%$. It occurred more commonly in women (65.6\%) than men.
\end{abstract}

\section{Introduction}

An estimated 422 million adults are living with diabetes mellitus globally, according to World Health Organization latest data 2016 (WHO) ${ }^{[1]}$. Prevalence of diabetes in adults in India is $8.8 \% .^{[2]}$ Diabetes is a systemic disease which affects almost all organ systems. Apart from causing major macro \& microvascular complications Nonalcoholic fatty liver disease (NAFLD) is being increasingly recognized today as a potentially serious complication of diabetes, especially type 2. NAFLD is defined as hepatic steatosis diagnosed either by histology/imaging with macrovesicular steatosis in $>5 \%$ of hepatocytes according to histological analysis or by proton density fat fraction or $>5.6 \%$ as assessed by proton magnetic resonance spectroscopy (MRS) or quantitative fat/water selective magnetic resonance imaging (MRI) with no secondary cause for steatosis. ${ }^{[3]}$ The spectrum of Non- 
alcoholic fatty liver disease (NAFLD) encompasses simple steatosis or steatosis with mild inflammation to severe non-alcoholic steatohepatitis (NASH).

The high incidence of non-alcoholic steatohepatitis (NASH) in patients with T2DM leads to further complications, such as liver cirrhosis and hepatocellular carcinoma, which are increasingly recognized. ${ }^{[4]}$

The Risk Factors for Nonalcoholic Fatty Liver Disease include:

Type 2 diabetes mellitus, Metabolic syndrome ${ }^{[5]}$, Obesity $^{[5]}$, Physical inactivity ${ }^{[6]}$, A high-calorie diet, excess saturated fats, refined carbohydrates, sugar-sweetened beverages, a high fructose intake $^{[3]}$ and Obstructive sleep apnea. ${ }^{[3]}$ NAFLD is usually slowly progressive; but in 20\%, it progresses rapidly. ${ }^{[7]}$ Progression in NAFL to fibrosis Stage 1 is every 14 years and every 7 years in NASH, which is further increased in the presence of arterial hypertension. ${ }^{[8]}$ Cirrhosis and liver failure occur in $11 \%-20 \%$ in NASH patients over $10-15$ years. $^{[8]}$

So it is of prime importance to screen for Nonalcoholic fatty liver disease (NAFLD) before it progresses to non-alcoholic steatohepatitis (NASH) especially in patients with risk factors like T2DM. ${ }^{(4)}$ T2DM patients manifest increased risk of Non-alcoholic fatty liver disease (NAFLD) both because of the high prevalence of obesity in T2DM and the effect of insulin resistance on fat metabolism. Early data suggest that some of the pharmacological agents commonly used in the management of blood glucose and dyslipidemia in T2DM may also be efficacious in treating Nonalcoholic fatty liver disease (NAFLD). T2DM patients thus represent a population that should be screened for Non-alcoholic fatty liver disease (NAFLD). Current study estimates the prevalence of Non-alcoholic fatty liver disease (NAFLD) among T2DM patients and also look for an association between lipid profile, central obesity, and presence of Non-alcoholic fatty liver disease (NAFLD).

\section{Materials and Methods}

This observational study was conducted at R L Jalappa Hospital on 75 diabetics (both type 1 and type 2).

Ethical clearance from the institution and written informed consent from the patient and attendees was taken.

The patient's weight and height were measured and BMI calculated. A BMI >25 was considered overweight.

Waist-hip ratio was also measured to look for central obesity.

If the ultrasound showed evidence of fatty liver, with or without the elevation of transaminases, a presumptive diagnosis of Non-alcoholic fatty liver disease (NAFLD) was made.

USG has a sensitivity of $84.8 \%$, specificity of $93.6 \%$, PPV of $77 \%$, and NPV of $67 \%$. $^{[3]}$

Transient elastography (TE) and MRI are also non-invasive imaging techniques.

For a precise diagnosis of NAFLD, liver biopsy is the investigation of choice ${ }^{[9]}$

Alcoholics, seropositivity to $\mathrm{HIV}, \mathrm{HCV}, \mathrm{HBsAg}$, ELISA and Patients on drugs that are proven to cause steatohepatitis (steroids, amiodarone, oral contraceptive pills, and other estrogen-containing preparations were excluded from the study.

Liver function test, Lipid profile, Ultrasound abdomen, FBS, PPBS, and HBA1C was done routinely.

Statistical analysis: Data analysis was performed using SPSS software (version 20) and Microsoft Excel worksheet 2016. Categorical variables were represented as proportions/percentages and quantitative variables were represented as means and standard deviation.

\section{Results}

Among the 75 diabetics who were studied, fatty liver was found in 32 patients $(42.66 \%)$.

\begin{tabular}{|c|c|}
\hline $\begin{array}{c}\text { No. of patients with } \\
\text { fatty liver }\end{array}$ & $\begin{array}{c}\text { No. of patients without fatty } \\
\text { liver }\end{array}$ \\
\hline $32(42.66 \%)$ & $43(57.33 \%)$ \\
\hline
\end{tabular}




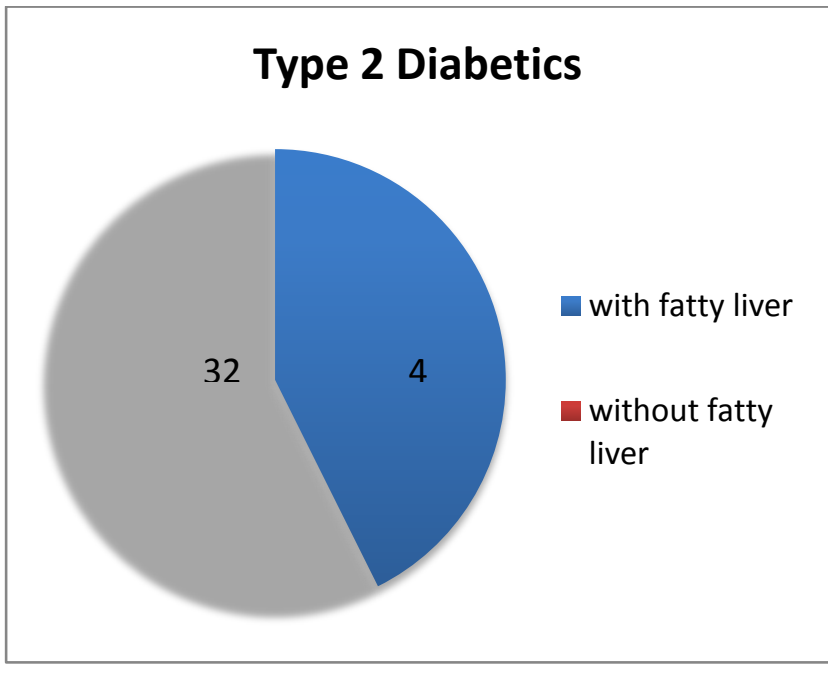

The number of males with fatty liver was 11 $(34.3 \%)$ and females 21(65.6\%)

\begin{tabular}{|l|c|c|}
\hline Sex & Fatty liver present & Fatty liver absent \\
\hline Male & 11 & 31 \\
\hline Female & 21 & 12 \\
\hline
\end{tabular}

The number of patients with fatty liver who had central obesity (waist-hip ratio >0.95) - 27 $(84.3 \%)$. All the patients with fatty liver had central obesity (waist-hip ratio $>0.95$ in males and $>0.85$ in females)

\begin{tabular}{|c|c|}
\hline Waist hip ratio $>0.95$ & Waist hip ratio $<0.95$ \\
\hline $27(84.3 \%)$ & $5(15.6 \%)$ \\
\hline
\end{tabular}

The number of patients who had increased triglycerides $>150$ among patients with fatty liver $26(81.2 \%)$

\begin{tabular}{|c|c|}
\hline $\begin{array}{c}\text { No. of patients } \\
\text { with } \uparrow \text { TGL }\end{array}$ & $\begin{array}{c}\text { No. of patients } \\
\text { with normal TGL }\end{array}$ \\
\hline 26 & 6 \\
\hline
\end{tabular}

No. of patients who were overweight among the persons detected to have fatty liver $-25(78.1 \%)$ No. of patients with normal BMI among the persons with fatty liver $-7(21.8 \%)$

\begin{tabular}{|c|c|}
\hline $\begin{array}{l}\text { No. of patients } \\
\text { with BMI }>25\end{array}$ & $\begin{array}{l}\text { No. of patients } \\
\text { with BMI }<25\end{array}$ \\
\hline 25 & 7 \\
\hline
\end{tabular}

No. of patients with increased cholesterol (>200 $\mathrm{mg} \%$ ) among patients with fatty liver -23 $(71.8 \%)$

\begin{tabular}{|c|c|}
\hline $\begin{array}{c}\text { Patients with } \\
\text { cholesterol }>200\end{array}$ & $\begin{array}{c}\text { Patients with cholesterol } \\
<200\end{array}$ \\
\hline $23(71.8 \%)$ & $9(28.1 \%)$ \\
\hline
\end{tabular}

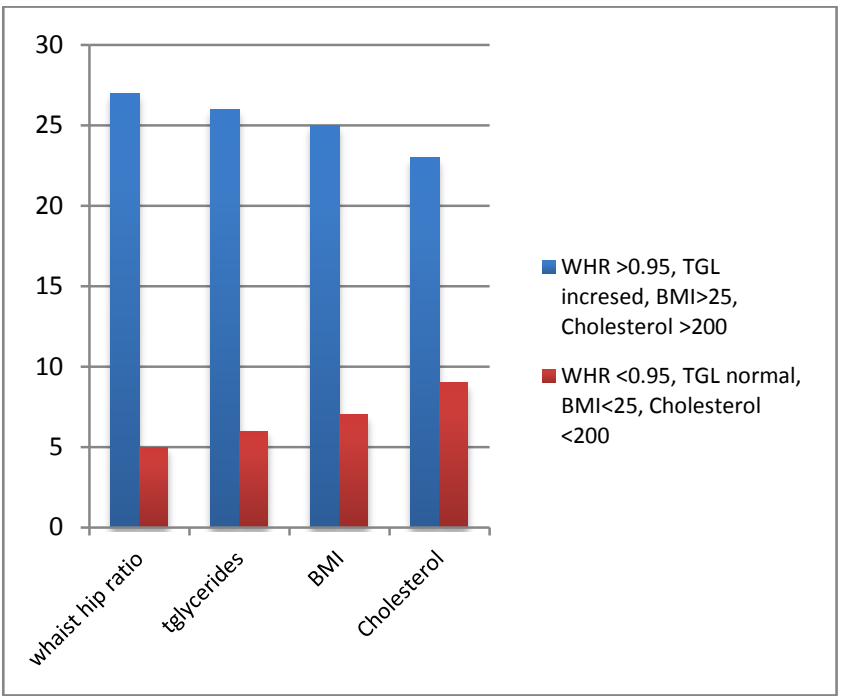

All the patients with ultrasound evidence of fatty liver showed marginally elevated transaminases and occasionally of serum alkaline phosphatase. There was no alteration in the serum protein or albumin globulin ratio.

\section{Discussion}

The prevalence of fatty liver amongst Type 2 diabetics in our study was found to be $42.66 \%$. According to a study conducted by Daad $\mathrm{H}$. Akbar, the prevalence of NAFLD was found to be $55 \% .^{[10]}$ The overall prevalence of NAFLD in patients with type 2 diabetes ranges from $40 \%$ to $70 \% .^{[7]}$

Among the patients with NAFLD, the percentage of patients who were overweight was $78.1 \%$. Wanless and Lentz found mild to severe steatosis in approximately $70 \%$ of obese patients and $35 \%$ of lean patients. ${ }^{[11]}$

Garcia Monzon et al found NASH in $69 \%$ whereas $22 \%$ had simple steatosis and only $8 \%$ had normal biopsy findings. ${ }^{[12]}$ In another study, the presence of NAFLD was highest among obese patients, with BMI of $30+/-5.5 \mathrm{~kg} / \mathrm{m} 2$ ${ }^{[13]}$ However, in another study, there was no significant difference in body mass index among patients with NAFLD. ${ }^{[10]}$

NASH can exist with only nonspecific symptoms for years in obese patients before manifesting itself either incidentally or with complications of cirrhosis or portal hypertension.

The prevalence of fatty liver was found to be higher among women (65.6\%) than men. Many 
studies have found the presence of fatty liver to be higher in women. ${ }^{[10,14]}$

NAFLD and NASH have been described in patients without the classic risk factors of obesity, diabetes, and overt hyperlipidemia. It has been described in patients with central or visceral adiposity in a study conducted by Bacon BR et al. ${ }^{[12]}$ In another study, fatty liver was strongly correlated with visceral adipose tissue. ${ }^{[10]}$ In this study, the percentage of patients with central obesity among patients with fatty liver was $100 \%$ and those with a waist-hip ratio of $>0.95$ in men and $>0.85$ in women was $84.3 \%$.

The no. of patients with increased triglycerides and cholesterol was found to be $81.2 \%$ and $71.8 \%$ respectively. As mentioned earlier two-thirds of patients with hypertriglyceridemia and one-third of patients with hypercholesterolemia have fatty liver. ${ }^{[15]}$ In another study, among patients with obesity and fatty liver, approximately $20 \%$ have some type of previously identified hyperlipidemia. ${ }^{[10]}$ In other studies, fatty liver was strongly correlated with the degree of dyslipidemia, especially the level of triglycerides. Hypertriglyceridemia was identified as an important risk factor in the development of NAFLD and NASH, it also correlates well with the histological severity of the disease. ${ }^{[10.12 .13]}$

\section{Conclusion}

The prevalence of non-alcoholic fatty liver disease in type 2 diabetes mellitus was $42.6 \%$. It occurred more commonly in women $(65.6 \%)$ than men.

The occurrence of the non-alcoholic fatty liver was found to be higher in patients who were overweight/ obese and in those with central obesity. $81.2 \%$ of the patients with fatty liver had dyslipidemias (especially hypertriglyceridemia).

\section{References}

1. WHO: Projections of global mortality and burden of disease from 2002 to 2030 . Mathers CD, Loncar D. PLoS Med, 2006, 3(11):e442.

2. Members [Internet].Idf.org. 2018 [cited 22
October 2018]. Available from: https://www.idf.org/our-network/regionsmembers/south-east-asia/members/94india

3. European Association for the Study of the Liver (EASL), European Association for the Study of Diabetes (EASD), European Association for the Study of Obesity (EASO). EASL-EASD-EASO clinical practice guidelines for the management of the non-alcoholic fatty liver disease. $\mathbf{J}$ Hepatol 2016;64:1388-402.

4. Tilg H, Moschen A, Roden M. NAFLD and diabetes mellitus. Nature Reviews Gastroenterology 2016;14(1):32-42.

\&Hepatology.

5. Hillenbrand A, Kiebler B, Schwab C, Scheja L, Xu P, Henne-Bruns D, et al. Prevalence of non-alcoholic fatty liver disease in four different weight-related patient groups: Association with small bowel length and risk factors. BMC Res Notes 2015;8:290.

6. Ryu S, Chang Y, Jung HS, Yun KE, Kwon MJ, Choi Y, et al. Relationship of sitting time and physical activity with the nonalcoholic fatty liver disease. J Hepatol 2015;63:1229-37.

7. Anstee QM, McPherson S, Day CP. How big a problem is the non-alcoholic fatty liver disease? BMJ 2011;343:d3897.

8. Singh S, Allen AM, Wang Z, Prokop LJ, Murad MH, Loomba R, et al. Fibrosis progression in nonalcoholic fatty liver vs. nonalcoholic steatohepatitis: A systematic review and meta-analysis of paired-biopsy studies. Clin Gastroenterol Hepatol 2015; 13:643-540.

9. Hazlehurst JM, Woods C, Marjot T, Cobbold JF, Tomlinson JW. Non-alcoholic fatty liver disease and diabetes. Metabolism 2016;65:1096-108.

10. Daad H. Akbar, Abeer H. Kawther. Diabetes Care 26:3351-3352, 2003.

11. Wanless IR, Lentz JS. Fatty liver hepatitis 
(steatohepatitis) and obesity: An autopsy study with analysis of risk factors. Hepatology 1990;12: 1106 -1110.

12. Garcia Monzon C, Martin Perez E, Lo Iacono $\mathrm{O}$ et al. Characterization of pathogenic and prognostic factors of nonalcoholic steatohepatitis associated with obesity. J Hepatol 2000;33:716-724.

13. Gupta P, Amarapukar D, Agal S, et al. Non-alcoholic steatohepatitis in type 2 diabetes mellitus. J Gastroenterol Hepatol. 2004 Aug;19(8):854-8.

14. Stephen H Caldwell, Tri H Le, Stacey M Anderson. Schiff's diseases of the Liver, 9th ed, edited by Eugene Schiff, Michael F Sorrell, Willis C Maddrey. Lippincott Williams and Wilkins pub, 2003. 1261 1280.

15. Assy N, Kaita K, Mymin D et al. Fatty infiltration of liver in hyperlipidemic patients. Dig Dis Sci 2000;45:1929-1934.

16. Dai W, Ye L, Liu A, Wen S, Deng J, Wu $X$ et al. Prevalence of nonalcoholic fatty liver disease in patients with type 2 diabetes mellitus. Medicine. 2017;96 (39) :e8179.

17. Wilner IR, Waters B, Patil SR et al. Ninety patients with alcoholic steatohepatitis: Insulin resistance, familial tendencies, and severity. Am J Gastroenterol 2017;96: 2957-2961.

18. Day CP, James OFW. Steatohepatitis: a tale of two hits. Gastroenterology 2016;114:842-845.

19. Sayiner M, Koenig A, Henry L, Younossi ZM. Epidemiology of Nonalcoholic Fatty Liver Disease and Nonalcoholic Steatohepatitis in the United States and the Rest of the World. Clin Liver Dis. 2016;20:205-214. 